\title{
Maze-arm length affects a choice criterion in the radial-arm maze
}

\author{
MICHAEL F. BROWN and CYNTHIA K. HUGGINS \\ Villanova University, Villanova, Pennsylvania
}

\begin{abstract}
Male rats were tested in a 12-arm radial maze with 6 arms that were standard in length and 6 arms that were half the standard length. As previously reported by Brown (1990), revisits to short arms were more likely than revisits to long arms. Two explanations of this effect of mazearm length on choice accuracy were experimentally contrasted. The first attributes the effect to diminished discriminability of visited and unvisited arms when the arms are short. The second attributes the effect to a relatively lax choice criterion being applied to short arms. An analysis of the microstructure of choices, applying the logic of signal detection theory, provided evidence for the latter explanation.
\end{abstract}

Memory performance in humans has been characterized in terms analogous to the signal detection theory (SDT) view of detection performance (e.g., Banks, 1970; Lockhart \& Murdock, 1970). That is, memory performance can be understood as a joint product of factors affecting the discriminability of an item as having been part of a to-be-remembered set $\left(d^{\prime}\right)$ and other (nonmemorial) factors affecting the tendency of subjects to classify an item as having been in the set $(\beta)$. Recently, a number of researchers concerned with discrimination, choice, and memory performance in animal subjects have adopted this general approach (Commons, Nevin, \& Davison, 1991).

The radial-arm maze (Olton \& Samuelson, 1976) has come to be a dominant spatial paradigm in the psychological literature concerned with animal memory. Relatively little work, however, has focused on the role of processes other than memory in radial-arm-maze performance. The present article is concerned with the role of choice-criterion effects on performance in this task. In this context, choice-criterion effects are factors other than the visited versus unvisited status of the maze arm that influence the tendency of the rat to visit an arm.

Brown, Wheeler, and Riley (1989) proposed that choice in the radial-arm maze can be described in terms of two separable processes corresponding to the two general types of processes identified by SDT. The first is the ability of rats to discriminate previously visited maze arms from those that have not yet been visited. This ability is presumably based on memories resulting from arm visits. The second process determining choice accuracy, according to Brown et al.'s model, is the choice criterion applied to maze arms. Specifically, a relatively strict choice criterion can result in higher levels of choice accuracy.

This research was supported by National Institute of Mental Health Grant MH45004. Correspondence should be addressed to M. F. Brown, Department of Psychology, Villanova University, Villanova, PA 19085 (e-mail: brownmf@vuvaxcom.bitnet).
This is essentially because a rat that is relatively more likely to reject maze arms (i.e., investigate them but not visit them) is more likely to subsequently encounter (and then visit) a correct (previously unvisited) arm. Thus, choice-criterion levels and discriminability levels jointly determine choice accuracy in the radial-arm maze.

Brown (1990) reported that the length of maze arms in the radial-arm maze has a substantial effect on performance. Rats trained in a maze with arms approximately half the standard length demonstrated either lower levels of choice accuracy or higher levels of adjacent arm responding than did rats trained in a standard radial-arm maze. Adjacent arm responding consists of patterned responding in which the rat visits an arm that is spatially adjacent to the previously chosen arm, thereby allowing accurate choices without the necessity of discriminating previously visited arms from those not yet visited.

Brown (1990, Experiment 3) described a preliminary experiment designed to determine whether the effect of maze-arm length on radial-arm-maze performance was a function of differences in discrimination ability or a choice criterion. Lower levels of performance in mazes with short arms might be due to a lower ability of rats to discriminate previously visited arms from unvisited arms. Several factors can be identified as potential causes of such a lowered discriminability, including the smaller distance between the ends of the arms, which increases the similarity of the views of extra-maze cues seen from the arms. Alternatively, the effect of arm length on choice accuracy might be due to the application of a lax choice criterion to short maze arms relative to long maze arms.

Brown's (1990) experiment involved the use of a 16arm maze with 8 short arms and 8 long arms. Two findings favored the choice-criterion explanation over the discrimination-ability explanation. First, there was a tendency for the short maze arms to be visited early in the choice sequence. This effect is consistent with a difference in the choice criterion applied to long and short maze arms and, therefore, increases the plausibility of the view 
that a criterion difference accounts for differences in choice accuracy.

Second, rats were given two-alternative forced-choice (2AFC) tests in which a choice was made between two short arms (one of which was previously visited) or between two long arms (one of which was previously visited). If there was a difference in the ability of rats to discriminate visited from unvisited arms depending on arm length, then a difference in choice accuracy on long-arm tests and short-arm tests would be expected. Extensions of the theoretical work described in Brown et al. (1989) show that the application of a stricter choice criterion to long arms than to short arms may or may not result in higher levels of choice accuracy to long arms in 2AFC tests, depending on the level of discriminability $\left(d^{\prime}\right)$ in relation to the magnitude of the choice-criterion difference. Thus, the logic of the 2AFC test is asymmetrical in that whereas a difference in choice accuracy to short and long arms in the 2AFC test could be explained either in terms of discriminability or in terms of a choice-criterion difference, the lack of a difference is compatible only with the latter view. Brown (1990) found no statistically reliable difference in choice accuracy on 2AFC tests with short and long arms, thereby discrediting the discrimination view. However, interpretation of these findings is rendered somewhat ambiguous by the fact that a nonreliable difference in the direction predicted by the discrimination view was obtained. Thus, although Brown's (1990) results are most compatible with the view that a choice-criterion effect is responsible for the lower levels of choice accuracy to short maze arms, they are by no means definitive.

The present experiment was intended to further explore the possibility that a difference in the choice criterion applied to long and short maze arms exists and that such a difference accounts for lower choice accuracy to short arms than to long arms. Rats were trained in a 12-arm radial maze with 6 short arms and 6 long arms. To conform with the previous procedure of Brown (1990), the spatial location of the short and long arms was fixed, and arms of the two lengths were different in terms of brightness (the long arms were painted black, and the short arms were painted white). This was intended to facilitate the ability of the rats to discriminate between long and short arms. Given the widely known "preference" of rats for black over white, the choice of brightness assignments to long and short arms was designed to work against our expectation that lower levels of choice accuracy would be obtained with short (white) arms than with long (black) arms.

Two predictions of the view that a lax choice criterion is applied to short maze arms were tested. In a free-choice procedure, the relative probability of visiting short and long maze arms was measured. It was expected that there would be a bias for visiting short maze arms early in the choice sequence, as reported in a 16-arm maze by Brown (1990). Such a bias is predicted by the theory that a relatively lax choice criterion is applied to short maze arms and, as indicated above, increases the plausibility of the criterion-difference account of lowered choice accuracy to short arms.
In a forced-choice procedure, rats first visited a randomly chosen set of three short arms and three long arms. After a 15-min retention interval, they were allowed to complete the maze. The behavior of the rats in the central arena of the maze was videotaped, and the choice behavior of the rats was coded from the videotapes in terms of microchoices (Brown, 1992; Brown et al., 1989). A microchoice is an instance of visual investigation of an individual maze arm, which terminates either in an arm visit (macrochoice) or in rejection of the arm. Microchoice outcomes can be categorized as hits (correct macrochoices), false alarms (incorrect macrochoices), misses (incorrect rejections), or correct rejections. Recent theories of decision processes in the context of rat radial-arm-maze performance (Brown, in press) and pigeon matching-to-sample performance (Wright, 1991) have successfully used this approach to describe and explain choice behavior.

If a relatively lax criterion is applied to short maze arms, then higher hit rates and/or lower correct rejection rates should occur to short maze arms than to long maze arms. In SDT terms, this pattern of results will produce a difference in $\log \beta$, the standard measure of choice criterion (e.g., Macmillan \& Creelman, 1991; McNicol, 1972). On the other hand, if visited arms and unvisited arms are less discriminable when arms are short, then both lower hit rates and lower correct rejection rates (lower levels of $d^{\prime}$, the standard measure of discriminability) should be obtained with short arms than with long arms. Thus, this technique allows discrimination and choice-criterion interpretations of the effects of maze-arm length on choice accuracy to be directly contrasted.

\section{METHOD}

\section{Subjects}

The subjects were 16 male Sprague-Dawley rats obtained as weanlings from Harlan Sprague Dawley, Inc. They were experimentally naive and approximately 4 months old at the beginning of this experiment. Their diet was restricted to $13 \mathrm{~g}$ of Purina Rat Chow daily for 2 weeks prior to the introduction to the experimental procedures and throughout the experiment. They were housed in groups of 3 or 4 and were transported daily between the colony and laboratory. Experimental sessions were conducted during the dark phase of a 12:12-h light:dark cycle.

\section{Apparatus}

The apparatus was a 12 -arm radial maze constructed of $1.5-\mathrm{cm}$ thick plywood. The circular central arena of the maze was $60 \mathrm{~cm}$ in diameter. The arms were $10 \mathrm{~cm}$ wide. If the 12 arms are considered to be numbered consecutively in a clockwise fashion, then arm numbers $1,2,3,6,8$, and 9 were $80 \mathrm{~cm}$ in length, and the remaining arms were $40 \mathrm{~cm}$ in length. A square black food cup $(4.5 \mathrm{~cm} \times 4.5 \mathrm{~cm} \times 0.7 \mathrm{~cm}$ deep) was located at the end of each arm. A wooden wall $(11 \mathrm{~cm}$ high $\times 18.5 \mathrm{~cm}$ long) was attached to one edge of each arm to prevent the rats from jumping from arm to arm without returning to the central arena. The aforementioned parts of the maze were painted white, with the exception of the surfaces of the long arms, which were painted flat black.

A metal panel was located at the entrance to each arm. Each panel had a 7.8-cm-diam hole that allowed access to the maze arm. The hole could be covered with a metal door, which slid in a vertical track and was controlled by the experimenter using a string and pulley system. This allowed access to maze arms to be individu- 
ally controlled. A clear Plexiglas wall surrounded the central arena. It included $7.8-\mathrm{cm}$ holes coinciding with those in the metal panels.

The maze was elevated $57 \mathrm{~cm}$ above the floor of the experimental room. The room was $4.4 \times 3.1 \mathrm{~m}$ and contained a number of salient extra-maze stimuli. It was illuminated by two fluorescent tubes. A convex mirror was located above the maze and was used to record behavior via a video camera.

\section{Procedure}

During the first 3 days of exposure to the experimental apparatus, the rats were placed in the apparatus in groups of 3 or 4 (cagemates). Forty-five-milligram sucrose pellets (BioServe, Inc.) were located in the food cups, on the arms of the maze, and in the central arena. The rats were left in the apparatus until all the pellets were consumed.

Beginning on Day 4 , the rats were run individually in a free-choice procedure for one session per day. Prior to each session, two pellets were placed in each food cup. The doors to all 12 arms were open throughout this phase of the experiment. The rat was placed in the central arena and allowed to choose arms until all 12 arms had been visited, 24 choices had been made, or $12 \mathrm{~min}$ had elapsed. A choice was defined as the rat's nose crossing the plane defined by the end of the wall along the edge of the arm (i.e., $18.5 \mathrm{~cm}$ from the central arena). The experimenter recorded the sequence of arms chosen. The rats were run in this free-choice procedure until 50 successful sessions were completed (i.e., all 12 arms were visited within 12 min).

The rats were then tested using a forced-choice procedure. A randomly ordered sequence of 3 short arms and 3 long arms was chosen for each session. These arms were baited. The rat was placed in the central arena with the doors to all 12 maze arms closed. After approximately $5 \mathrm{sec}$, the door to the first arm in the sequence was opened. While the rat visited that arm, the door to the second arm was opened. Thereafter, as the rat visited each arm in the sequence, the door to the previous arm was closed and the door to the next arm was opened. Following its return to the center after the 6th choice, the rat was removed from the maze and placed in an individual cage, distinct from the home cage in size and construction, for $15 \mathrm{~min}$.

After this delay, the rat was returned to the central arena. All maze-arm doors were open, but only the six unvisited arms were baited. The rat was allowed to make choices until all baited arms were visited or until 5 min elapsed without a choice. The behavior of the rat was videotaped. This procedure was continued for 25 sessions, not including sessions during which the 5-min time limit occurred.

Videotapes of postdelay behavior were coded by two coders, both of whom were naive to the theoretical issues under investigation. One coder (Coder A) coded the data from all 25 sessions. A second coder (Coder B) coded data from the last 10 sessions in order to allow measurement of interrater reliability. A microchoice was defined for the coders as "clear orientation toward the end of an arm that was accompanied either by a discernable stop in the motion of the rat or by a macrochoice." Each coder independently identified the sequence of arms to which a microchoice was directed and whether the microchoice ended in an arm visit (macrochoice) or a rejection. The hit rate and correct rejection rate to long and short arms were determined from the data coded by Coder $\mathrm{A}$.

\section{RESULTS}

\section{Free-Choice Phase}

Data from the last 25 sessions of the free-choice phase were analyzed. During these sessions, the rats required a mean of 13.6 choices to complete the maze. Figure 1 shows the percentage of choices that were made to short

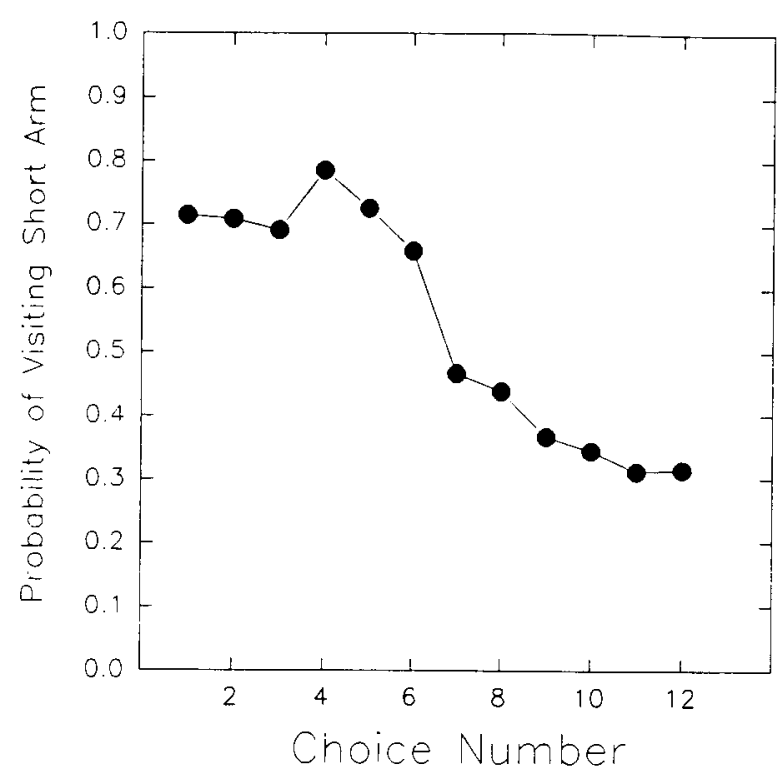

Figure 1. The probability of a visit to a short arm as a function of choice number during the free-choice phase.

arms over the course of the first 12 choices. An analysis of variance (ANOVA) revealed a reliable effect of choice number $[F(11,165)=14.2, p<.001]$. During early choices, there was a clear preference for short maze arms over long maze arms.

Figure 2 shows the probability of revisiting long and short arms as a function of the ordinal position of the initial choice among correct choices. An ANOVA (arm length $\times$ ordinal position) revealed an effect of ordinal position $[F(11,165)=9.5, p<.001]$. There was no significant main effect of arm length $[F(1,15)=1.7]$. However, the effects of these two variables produced a significant interaction $[F(11,165)=1.9, p<.05]$. This interaction indicates that an effect of arm length on the probability of revisiting a maze arm was restricted to choices made early in the choice sequence.

\section{Forced-Choice Phase}

One rat failed to perform following the introduction of the forced-choice procedure and was therefore dropped from the experiment. A 2 nd rat died of unknown causes during the forced-choice phase. The data from these 2 rats are not included in the following analyses.

The remaining rats required a mean of 8.3 postdelay choices to complete the maze during the forced-choice phase. The source of the 2.3 errors made included a mean of 1.0 error per session to long arms that were in the set of predelay arms and 1.2 errors per session to short arms that were in the set of predelay arms. This difference is marginally reliable $[F(1,13)=3.85, p=.07]$.

Two rats were eliminated from the microchoice analysis because they failed to show sufficient levels of arm rejection. Over the course of the 25 sessions, 1 of these rats rejected maze arms on 7 occasions and the other re- 


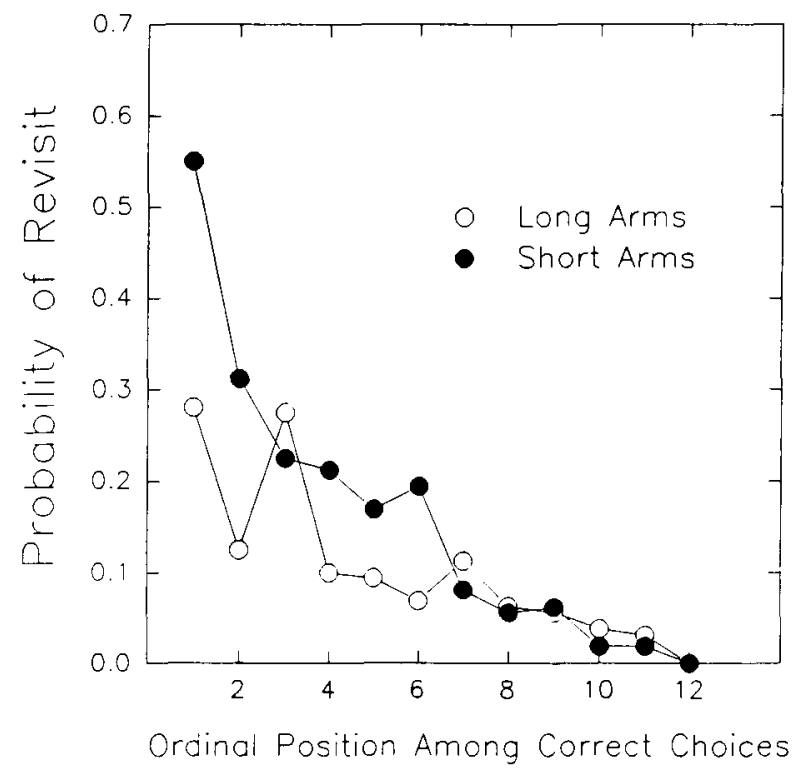

Figure 2. The probability of a revisit to an arm as a function of arm length and the ordinal position of the original correct visit to the arm during the free-choice phase.

jected maze arms on 21 occasions. The remaining rats showed much higher levels of arm-rejection behavior (range: 146-300 instarces). Interrater reliability of the coded microchoice data was determined only for microchoices resulting in arm rejections (because there is no ambiguity in microchoices resulting in an arm visit). A mean (over rats) of $90.8 \%$ of the arm rejections coded by Coder $\mathrm{A}$ were also coded by Coder $\mathrm{B}$.

Figure 3 shows the mean probability of correctly rejecting previously visited long and short arms and of correctly visiting long and short arms, as revealed by the analysis of microchoices. The hit and correct rejection rates of individual rats were used to determine values of $d^{\prime}$ and $\log \beta$ for each arm length. Mean $d^{\prime}$ for long arms was 1.38, and mean $d^{\prime}$ for short arms was 1.35 . These values do not differ $[F(1,11)<1]$. The criterion value $(\log \beta)$ for long arms (.16) was reliably different from the criterion value for short arms $(.07)[F(1,11)=5.04, p<.05]$. The criterion values indicate that a bias for rejecting arms over visiting arms was stronger in the case of long arms than in the case of short arms.

\section{DISCUSSION}

Brown (1990) reported low levels of accuracy in both 16-arm and 12-arm radial mazes with short arms, as well as a direct comparison of choice accuracy in a 12-arm maze with short arms and a 12-arm maze with long arms. In the latter case, clear evidence for lower levels of choice accuracy in a maze with short arms was found. The results of the present experiment replicate the basic effect of maze-arm length on choice accuracy, although the mag- nitude of the effect appears to be lower in the present experiment than in the experiments of Brown (1990). In the free-choice phase, there was a higher probability of revisiting short arms chosen early in the choice sequence than of revisiting long arms chosen early in the choice sequence. There also appeared to be a higher probability of revisits to short arms than to long arms in the forcedchoice procedure. Although the latter effect did not quite reach the standard criterion of statistical reliability, the two results considered together and in the context of the results reported by Brown (1990) provide consistent support for the view that choice accuracy is generally lower with shorter maze arms than with longer maze arms.

It should be noted that this effect is obtained despite the difference in brightness in the short and long maze arms, which may work against the effect. Recent data collected in our laboratory (Brown \& Lesniak-Karpiak, in press) indicate that the use of a black surface on the long arms and a white surface on the short arms does, in fact, reduce the magnitude of the effect of arm length on choice accuracy, possibly because of a lower choice criterion to black arms than to white arms. Whether the difference in brightness is necessary or important in allowing the rats to discriminate short and long maze arms (its intended function) is unknown.

Two results from the present experiment support the hypothesis that a more lax choice criterion is applied to short maze arms than to long maze arms. First, there was a strong tendency for short arms to be visited early in the choice sequence. This result indicates a difference in the choice criterion applied to long and short maze arms and, therefore, increases the plausibility of the view that the

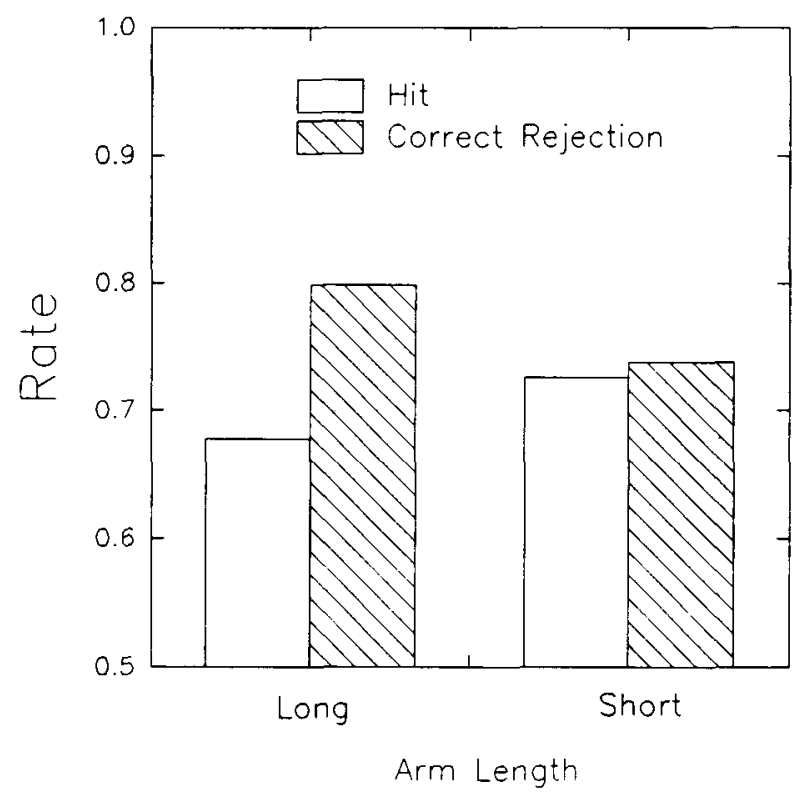

Figure 3. The outcome of microchoices to previously unvisited arms (hit rate) and previously visited arms (correct rejection rate) as a function of arm length during the forced-choice phase. 
difference in accuracy as a function of maze-arm length is mediated by a choice-criterion difference.

Second, and most critically, choice behavior during a forced-choice procedure was analyzed in terms of go/nogo decisions regarding individual maze arms, which are classified in terms of signal detection theory. There was a stronger tendency for rats to reject long arms than to reject short arms, indicating a relatively lax choice criterion for short maze arms. On the other hand, there was no evidence for a difference in the ability of rats to discriminate visited and unvisited arms as a function of arm length. This result provides direct evidence for the view that a choice-criterion difference, but not a discriminability difference, is produced by the difference in mazearm length.

Thus, the differences in memory performance for short and long maze arms reported by Brown (1990) and in the present article are apparently not mediated by a diminished ability on the part of rats to discriminate visited arms from unvisited arms when the arms are short. Instead, the difference in memory performance is attributable to a relatively higher tendency for rats to visit short arms that is independent of memory for those visits. In other words, rats are more likely to revisit short arms because they are more likely to visit short arms.

The effect of maze-arm length on choice criterion can be interpreted in a number of ways. One possibility is the increased effort required for reinforcement of visits to long arms relative to visits to short arms. The relationship between effort and reinforcement as a determinant of choice in discrimination performance has a long history (e.g., Davison \& McCarthy, 1988), which could be applied to the effects reported in this article.

The role of effort has also been considered in the context of optimal foraging theory. Roberts and Ilersich (1989) placed barriers, over which rats climbed, on some of the arms of a four-arm radial maze in order to manipulate "travel time." They found that arm revisits were more likely when barriers were absent than when they were present. This result is analogous to the lowered levels of choice accuracy for short arms reported in the present article. Roberts and Ilersich interpreted their results in terms of the view that rats and other animals forage in a manner that is optimal (e.g., Stephens \& Krebs, 1986). Similarly, one could interpret the present results in terms of a tradeoff between the "cost" of arm visits and the "benefit" of obtaining food on the arms. The relatively low cost of visiting short arms would thereby result in a relatively high probability of such visits.

Yet another perspective on the present results comes from the extensive literature on the effects of delay to reinforcement (e.g., Commons, Mazur, Nevin, \& Rachlin, 1987). Clearly, the length of the maze arms affects the time that elapses between choice of the arm and ingestion of the pellets that are usually found at the end of the arm. This difference in delay to reinforcement could result in a difference in responding to long and short arms that is independent of memory for previous visits. Further experimentation will be required to choose among these possible explanations for the effect of arm length.

Regardless of the explanation for the effect of arm length on choice criterion, it underscores the need to consider nonmemorial factors in interpreting performance in this and other "memory" paradigms. The results also point the way toward separating the role of discrimination ability and criterion effects in the radial-arm maze. These techniques may allow future research to provide insights into the relationships among processes underlying discrimination ability and other variables influencing spatial performance.

\section{REFERENCES}

BANKs, W. P. (1970). Signal detection theory and human memory. Psychological Bulletin, 74, 81-99.

Brown, M. F. (1990). The effects of maze-arm length on performance in the radial-arm maze. Animal Learning \& Behavior, 18, 13-22.

Brown, M. F. (1992). Does a cognitive map guide choices in the radialarm maze? Journal of Experimental Psychology: Animal Behavior Processes, 18, 56-66.

Brown, M. F. (in press). Sequential and simultaneous choice processes in the radial-arm maze. In T. Zentall (Ed.), Animal cognition: A tribute to Donald A. Riley. Hillsdale, NJ: Erlbaum.

Brown, M. F., \& Lesniak-KarPiAK, K. B. (in press). Choice criterion effects in the radial-arm maze: Maze-arm incline and brightness. Learning \& Motivation.

Brown, M. F., Wheeler, E. A., Riley, D. A. (1989). Evidence for a shift in the choice criterion of rats in a 12 -arm radial maze. Animal Learning \& Behavior, 17, 12-20.

Commons, M. L., Mazur, J. E., Nevin, J. A., \&achlin, H. (1987). Quantitative analysis of behavior: Vol. 5. The effect of delay and of intervening events on reinforcement value. Hillsdale, $\mathrm{NJ}$ : Erlbaum.

Commons, M. L., Nevin, J. A., \& Davison, M. C. (1991). Signal detection: Mechanisms, models, and applications. Hillsdale, NJ: Erlbaum.

DAvison, M., \& MCCARTHY, D. (1988). The matching law: A research review. Hillsdale, NJ: Erlbaum.

LoCKHART, R. S., \& MURDOCK, B. B., JR. (1970). Memory and the theory of signal detection. Psychological Bulletin, 74, 100-109.

McNicol, D. (1972). A primer of signal detection theory. London: Allen \& Unwin.

Macmillan, N. A., Creelman, C. D. (1991). Detection theory: A user's guide. Cambridge: Cambridge University Press.

Olton, D. S., \& SAmUELson, R. J. (1976). Remembrance of places past: Spatial memory in rats. Journal of Experimental Psychology: Animal Behavior Processes, 2, 97-116.

RoBerts, W. A., \& ILERSICH, T. J. (1989). Foraging on the radial maze: The role of travel time, food accessibility, and the predictability of food location. Journal of Experimental Psychology: Animal Behavior Processes, 15, 274-285.

Stephens, D. W., \& Krebs, J. R. (1986). Foraging theory. Princeton, NJ: Princeton University Press.

Wright, A. A. (1991). A detection and decision process model of matching to sample. In M. L. Commons, J. A. Nevin, \& M. C. Davison (Eds.), Signal detection: Mechanisms, models, and applications (pp. 191-219). Hillsdale, NJ: Erlbaum.

(Manuscript received January 17, 1992; revision accepted for publication June 29,1992 .) 